\title{
Simulation and improvements of a magnetic flux sensor for application in immunomagnetic biosensing platforms
}

\author{
Kan Wang ${ }^{1}$, TangAn $\mathrm{Li}^{2}$, Bo $\mathrm{Cao}^{2}$, Hao $\mathrm{Xu}^{2}$, Wei Zheng ${ }^{2}$, YueMeng Cheng ${ }^{2}$, ChuJun \\ Zheng $^{2}$, Qinghui Jin ${ }^{3}$, and Da-Xiang Cui ${ }^{4}$ \\ ${ }^{1}$ Shanghai Jiao Tong Univ \\ ${ }^{2}$ Shanghai Jiao Tong University \\ ${ }^{3}$ Chinese Academy of Sciences \\ ${ }^{4}$ Shanghai JiaoTong university
}

June 26, 2021

\begin{abstract}
In recent years, point-of-care testing (POCT) has become a topical issue. Lateral flow immunoassay strategies based on magnetic nanoparticles (MNPs) are important POCT elements due to their sensitive quantification of biological materials via MNP magnetic field measurement. In this study, we designed a magnetic flux sensor for use in immunomagnetic biosensing platforms, incorporating a mathematical model and computer simulation strategy. The system used field programmable gate array (FPGA) as the control chip, synthesized excitation signals and excited coils to generate excitation magnetic fields. Also, the stepping motor was controlled to drive the test strip at a uniform speed through the sensor detection area. A differential configuration strategy was used for sensor pick-up coils to assess MNP influence on the magnetic flux, which was insensitive to background magnetic interference and common-mode noise. These factors significantly enhanced the signal-to-noise ratio of the sensor. The magnetic flux sensor structure was optimized, and response magnetic field characteristics of MNP on test strips analyzed using finite element analysis (FEA) simulations. System performance was evaluated by testing human chorionic gonadotropin (HCG), which demonstrated a linear performance, with a limit of detection of $0.0098 \mathrm{mIU} / \mathrm{mL}$. This system may be used to identify other target analytes in different application settings.
\end{abstract}

\section{Hosted file}

Manuscript-210621-Biotechnology and Bioengineering.docx available at https://authorea.com/ users/422198/articles/527931-simulation-and-improvements-of-a-magnetic-flux-sensor-forapplication-in-immunomagnetic-biosensing-platforms 


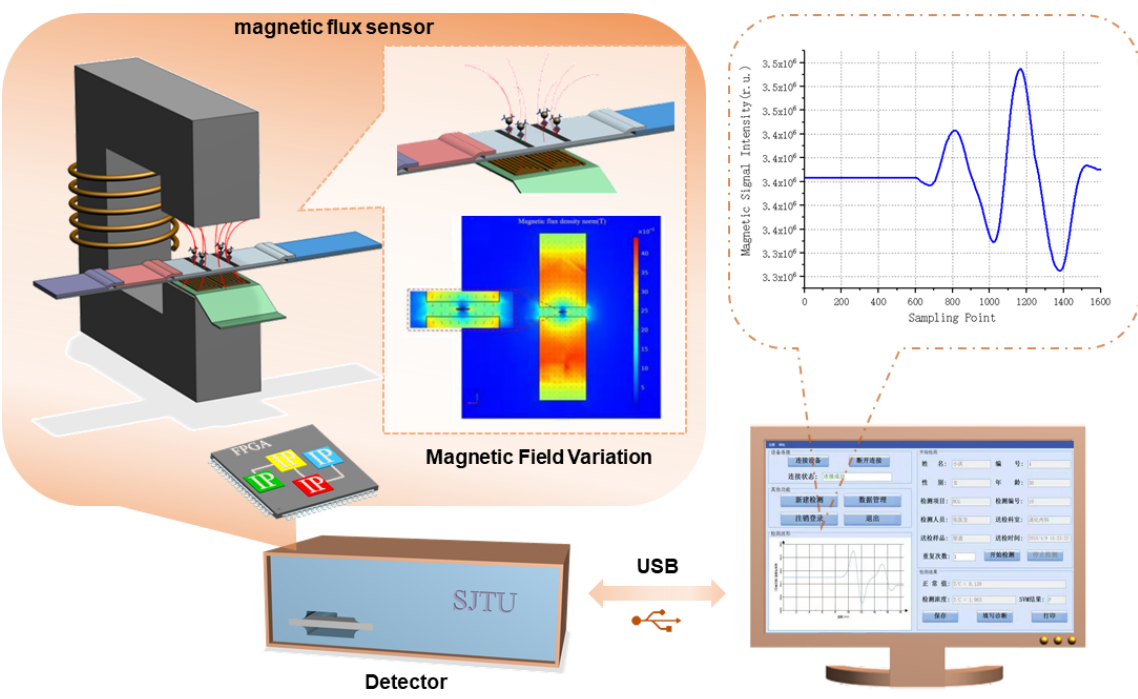

